\title{
Spectrum of AKI in Geriatric Population
}

\author{
Dr. Punit $\mathbf{G}^{1}$, Bharti $\mathrm{T}^{2}$, Dr. Nikita $\mathbf{J}^{3}$, Swati $\mathrm{S}^{4}$ \\ ${ }^{1}$ Professor Nephrology Unit, ${ }^{2}$ Fellowship in Advance Clinical Research, \\ ${ }^{3}$ Transplant Counselor, ${ }^{4}$ Physiotherapist \\ Department of Medicine, Dr. Bhimrao Ambedkar Hospital, Raipur, Chhattisgarh, India
}

\begin{abstract}
Introduction: Geriatric population is increasing worldwide due to increased life expectancy. Therefore the present study was conducted to see the spectrum of Acute Kidney Injury (AKI) in them. Acute kidney injury (AKI) is a new consensus term, encompassing a range of kidney diseases of acute onset. Two trials, namely program to improve care in acute renal disease (PICARD) and beginning and ending supportive therapy (BEST) for the kidney, confirmed that AKI is a significant contributor toward mortality, morbidity among ICU patients. RIFLE classification scheme and acute kidney injury network (AKIN) classification scheme have been proposed to achieve early diagnosis of AKI. A new consensus definition merging the criteria has also emerged from the Kidney Disease Improving Global Outcomes. With the application of these criteria, prevalence of AKI in ICU setting is $>40 \%$ if sepsis is present and the mortality rates varied from $15 \%$ to $60 \%$. Longer hospital stay and economic burden are inevitable. In contrast to western literature, few reliable statistics are available regarding AKI in India. We retrospectively evaluated patients with AKI, using the RIFLE criteria, to answer questions regarding most susceptible population, etiology, role of dialysis, outcomes and relation of mortality rate with RIFLE class.
\end{abstract}

Materials and Method: This retrospective study included 100 patients above the age of 50 years, in the Department of Medicine under nephrology unit, Dr. Bhimrao Ambedkar Hospital Raipur (C.G.), during the period of February 2018 - July 2018 were selected in this prospective study. Patients were analysed based on clinical presentation, biochemical, and sonographic parameters and classified under different categories of renal diseases.
Results: The mean age of patients was $56.62 \pm 5.44$ years. There were $65 \%$ males and $35 \%$ females. Chronic kidney disease was the commonest presentation, seen in $52 \%$ of patients. Acute kidney injury accounted for $38 \%$ whereas $10 \%$ of the patients had nephrotic syndrome. Sepsis and Acute gastroenteritis is the most common cause of AKI in the geriatric patients of our study.

Conclusion: Only very few studies are available on the spectrum of AKI in older age group. The incidence of AKI is increasing, especially among the elderly. Studies reveal that the elderly suffer higher morbidity and mortality from AKI.

Keyword: geriatric, elderly, acute kidney injury,

\section{Background}

Renal diseases are one of the common diseases causing high morbidity and mortality in otherwise asymptomatic individuals. The spectrum of renal diseases varies significantly in different parts of the world and is influenced by geographical, environmental and socioeconomic factors in that region. In addition, the spectrum also varies depending upon the population group being studied: community, outdoor patients or inpatients of a general/tertiary care hospital.

With increase in longevity, the older population is increasing worldwide. The older population is more prone to the deleterious effects of renal diseases, be it an acute insult to the normal renal functions or a chronic disease. In India, the older population accounts for $7.5 \%$ of the total population.

In spite of nephrology as a specialty since eighties, there is paucity of data regarding the spectrum of renal diseases in India. Available literatures from few 
hospitals across the country show data on specific clinical syndromes of renal diseases or specific renal diseases rather than the spectrum as a whole. In the studies we go through, the spectrums of acute kidney injury including chronic kidney diseases, glomerulonephritis, renovascular hypertension and renal amyloidosis. Therefore the present study has been planned with an aim to see the spectrum of acute kidney injury in geriatric patients admitted in the Department of Medicine under nephrology unit, Dr. Bhimrao Ambedkar Hospital Raipur (C.G.).

\section{Definition}

AKI was defined as patients whose serum creatinine and/or urine output fulfilled the RIFLE criteria. Risk class was defined as increase in serum creatinine $>1.5$ $\times$ baseline or urine output $<0.5 \mathrm{ml} / \mathrm{kg} / \mathrm{h}$ for the duration $>6 \mathrm{~h}$. Injury class was defined as increase in serum creatinine $>2.0 \times$ baseline or urine output $<0.5$ $\mathrm{ml} / \mathrm{kg} / \mathrm{h}$ for $>12 \mathrm{~h}$. Failure class was defined as increase in serum creatinine $>3.0 \times$ baseline or an absolute serum creatinine $4 \mathrm{mg} / \mathrm{dl}$ or urine output $<0.3$ $\mathrm{ml} / \mathrm{kg} / \mathrm{h}>24 \mathrm{~h}$ or anuria $>12 \mathrm{~h}$. Loss was defined as complete loss of kidney function $>4$ weeks, requiring dialysis. ESRD was defined as complete loss of kidney function, requiring dialysis for $>3$ months. Oliguria was defined as urine output below 500 $\mathrm{ml} /$ day. Patients who fulfilled RIFLE criteria within $48 \mathrm{~h}$ of admission were classified as community acquired AKI (CAAKI) and patients who fulfilled RIFLE criteria $48 \mathrm{~h}$ after admission were classified as hospital acquired AKI (HAAKI). Complete renal recovery was defined as estimated glomerular filtration rate (eGFR) returning to a value of $>60$ $\mathrm{ml} / \mathrm{min} / 1.73 \mathrm{~m} 2$ within 3 months. Chronic kidney disease (CKD) was defined as persistent reduction in eGFR after 3 months with a value $<60 \mathrm{ml} / \mathrm{min} / 1.73$ $\mathrm{m} 2$. Mortality was defined as patients expiring during the hospital stay.

\section{Criteria}

All patients aged above 50 years admitted to the Department of Medicine under nephrology unit, Dr. Bhimrao Ambedkar Hospital Raipur (C.G.) with AKI and those who developed AKI after admission to, during the period of Feb 2018 to July 2018, were included in our study. Patients with preexisting renal disease were also included in the study. A total of 100 patients met the above requirements and were evaluated retrospectively.

\section{Materials and Method}

The study was carried out patient admitted in the department of medicine under nephrology unit, Dr. Bhimrao Ambedkar Hospital Raipur (C.G.) between month of February to July 2018. The records of patients of more than 50 years of age, who presented at Kidney and Dialysis Clinic and were admitted at the department of medicine under nephrology unit, Dr. Bhimrao Ambedkar Hospital Raipur (C.G.) during the above mentioned period were analysed based on clinical presentation, biochemical, sonographic parameters. Ethical clearance has taken from the ethical committee of the Pt. JNM Medical College Raipur, prior to conduct the study. All participants and family members of the patients were provided written information consent.. The cut off age has been reduced to 50 years in this study to define older population due to variation in life expectancy in Indian population as compared to their western counterparts.

These patients were classified under various categories of renal diseases which included acute kidney injury (AKI), chronic kidney disease (CKD), nephrotic syndrome, obstructive uropathy and miscellaneous categories. These categories were further sub classified based on the available data.

\section{Result and Analysis}

A total of 100 older patients presented between Feb 2018 and Jul 2018, out of which $65(65 \%)$ were males and $35(35 \%)$ were females (Table 1). Mean age was $56.62 \pm 5.44$ years. CKD was the commonest presentation and was seen in $52 \%$ of the patients. AKI was the second most common presentation $38 \%$ followed by nephritic syndrome (Table 2).

Table (1) Demographic and clinical presentation of study patients

\begin{tabular}{|c|c|c|}
\hline Characteristics & (No.) & $(\%)$ \\
\hline Male & 65 & 65 \\
\hline Female & 35 & 35 \\
\hline
\end{tabular}

Table (2) Spectrum of Renal Diseases in study of older patients

\begin{tabular}{|c|c|c|}
\hline Characteristics & (No.) & $(\%)$ \\
\hline AKI & 38 & 38 \\
\hline CKD & 52 & 52 \\
\hline Nephrotic syndrome & 10 & 10 \\
\hline
\end{tabular}


International Journal of Trend in Scientific Research and Development (IJTSRD) ISSN: 2456-6470

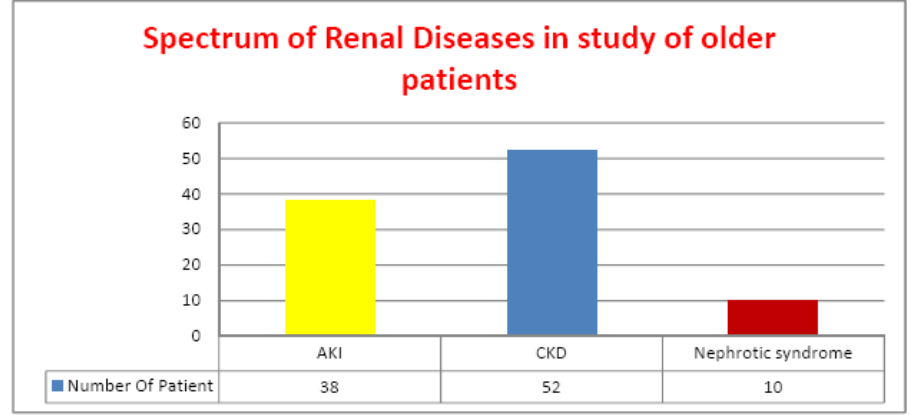

Graph (1): Spectrum of Renal Diseases in study of older patients

Most common cause of AKI was sepsis. Urinary tract infections were the most common source of sepsis, followed by respiratory infections and diabetic foot. Gastroenteritis was the second most common cause of AKI in our study. Obstetrical diseases were the third most common cause of AKI hepatic and cardiac disease constituted the other major causes. Drug induced AKI, snake bite, rapidly progressive glomerulonephritis (RPGN) were the other causes of AKI, although less common. Hair dye poisoning accounted for 3 cases, organophosphates accounted for two cases. The observations regarding the etiology of AKI in our study are summarized in (Table 3).

Table (3) Spectrum of Renal Diseases in study of older patients

\begin{tabular}{|c|c|c|}
\hline Etiology & $(\mathbf{N o})$ & $(\%)$ \\
\hline Sepsis & 45 & $45 \%$ \\
\hline Acute gastroenteritis & 18 & $18 \%$ \\
\hline Hepatic causes & 8 & $8 \%$ \\
\hline Cardiac causes & 8 & $8 \%$ \\
\hline Drug induced & 4 & $4 \%$ \\
\hline Snake Bite & 2 & $2 \%$ \\
\hline RPGN & 1 & $1 \%$ \\
\hline Other & 14 & $14 \%$ \\
\hline Total & 100 & $100 \%$ \\
\hline
\end{tabular}

Table (4) AKI distributing in relation to patient age Spectrum of Renal Diseases in study of older patients

\begin{tabular}{|c|c|c|}
\hline Age (Year) & (No.) & $(\%)$ \\
\hline $51-60$ & 44 & $44 \%$ \\
\hline $61-70$ & 32 & $32 \%$ \\
\hline $71-80$ & 15 & $15 \%$ \\
\hline$>80$ & 9 & $9 \%$ \\
\hline Total & 100 & \\
\hline
\end{tabular}

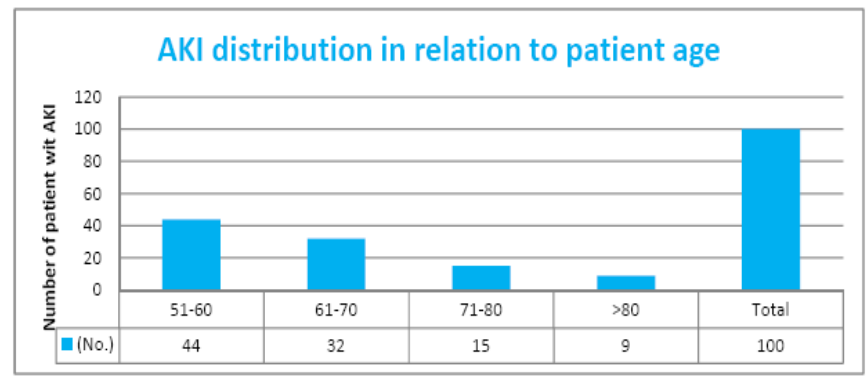

Graph (2): AKI distribution in relation to patient age Table (5) Mortality in our study group in relation to etiology

\begin{tabular}{|c|c|c|c|}
\hline Diagnosis & EExpired & Alive & Total (\%) \\
\hline Medical & 22 & 64 & 86 \\
\hline Sugical & 1 & 3 & 4 \\
\hline Obstetrical & 4 & 6 & 10 \\
\hline Total & 27 & 73 & 100 \\
\hline
\end{tabular}

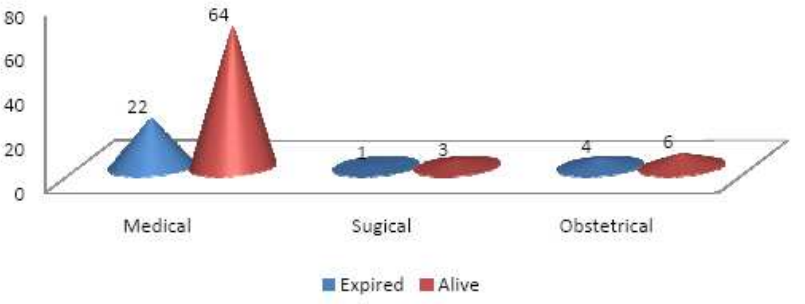

Graph (3): Mortality in relation to etiology

Table (5) Mortality in relation to risk, injury, loss, end stage class, failure (RILEF)

RILEF Class $\quad$ Number of Patient Expired (\%)

\begin{tabular}{|c|c|c|}
\hline Risk & 25 & $7(28)$ \\
\hline Injury & 36 & $10(27.77)$ \\
\hline Loss & 3 & $1(33.330$ \\
\hline End stage class & 1 & - \\
\hline Failure & 35 & $9(25.71)$ \\
\hline Total & 100 & $27(27)$ \\
\hline
\end{tabular}

\section{DISCUSSION}

With increasing life expectancy, proportion of older men and women suffering from renal disorders is on the rising trend. Greater proportions of older population are now seen occupying hospital beds with problems related to their kidneys.

Out of 100 older patients with renal diseases, CKD was the commonest presentation and was seen in 52 patients followed AKI seen in 38 patients. Nephrotic syndrome followed with 10 patients cases respectively.

AKI is becoming increasingly common in older people. Age-related changes in the renal and 
immunological system with the presence of multiple co morbidities render older patients more prone to renal injury. AKI is treated in the same way in older individuals and younger patients, older individuals are more vulnerable to dialysis related complications such as hemodynamic instability, bleeding, and mild disequilibrium syndrome.

In the present study Sepsis is the most common cause of AKI in the geriatric patients of our study. Age $>50$, male gender were prevalent in the majority of AKI patients. Crude mortality rate among patients with AKI in our study group was $37.6 \%$.

The Older are more prone to develop pre-renal AKI due to dehydration because of diminished fluid intake and impairment of the ageing kidneys to conserve sodium and water

\section{Conclusion}

The incidence of AKI is increasing, especially among the elderly. Anatomic and physiologic changes related to aging coupled with increased comorbidities appear to elevate the risk of developing AKI in older adults. A multitude of etiologies may cause or contribute to AKI and a careful assessment aided by serum, urinary, and radiologic tests will often arrive at the appropriate diagnosis. Studies reveal that the elderly suffer higher morbidity and mortality from AKI. However, reasonable outcomes are obtained in most elderly patients with AKI and age alone should not be a criterion for withholding supportive therapies. A standardized staging system for AKI coupled with a growing knowledge of its pathophysiology may allow for the identification of future treatments and consequent improvements in outcomes in the coming years.

\section{References}

1. Bellomo R, Kellum JA, Ronco C. Acute kidney injury.Lancet.2012; 380:756-66.

2. The Kidney Disease Improving Global Outcomes (KDIGO) Working Group. Definition and classification of acute kidney injury. Kidney Int.2012; 2(1 Suppl 2):19-36.

3. Case J, Khan S, Khalid R, Khan A. Epidemiology of acute kidney injury in the intensive care unit. Crit Care Res Pract 2013.2013:479730.

4. Kumar R, Hill CM, McGeown MG. Acute renal failure in the older. Lancet 1973; 1:90-1.

5. Barsoum RS. Malaria acute renal failure. J Am Soc Nephrol 2000; 11:2147-54. (PMID: 11053494).

6. Hsu Cy, McCulloch CE, Fan D, Ordoñez JD, Cher tow GM, Go AS. Community based incidence of acute renal failure. Kidney Int 2007; 72:208e12.

7. Ali T, Khan I, Simpson W, Prescott G, Townend J, Smith W, et al. Incidence and outcomes in acute kidney injury: a comprehensive population-based study.J Am Soc Nephrol 2007;18:1292e8.

8. Anderson S, Eldadah B, Halter JB, Hazzard WR, Himmelfarb J, Horne FM, et al. Acute kidney injury in older adults. J Am Soc Nephrol 2011; 22:28e 38 .

9. Chao CT, Wu VC, Lai CF, Shiao CC, Huang TM, Wu PC, et al. Advanced age affects the outcome predictive Power of RIFLE classification in geriatric patients with acute kidney injury. Kidney Int 2012; 82:920e7.

10. Glassock RJ, Rule AD. The implications of anatomical and functional changes of the aging kidney: with an emphasis on the glomeruli. Kidney Int 2012; 82: 270e7. 